\title{
Efficacy of Compression Gloves in Maintaining Edema Reductions After Application of Compression Bandaging to the Stroke-Affected Upper Limb
}

\author{
Louise Gustafsson, Elizabeth Patterson, Kathryn Marshall, \\ Sally Bennett, Kylie Bower
}

\section{MeSH TERMS}

- compression bandages

- edema

- hand

- stroke

- upper extremity
Louise Gustafsson, PhD, is Associate Professor, School of Health and Rehabilitation Sciences, The University of Queensland, St. Lucia, Brisbane, Queensland, Australia; I.gustafsson@uq.edu.au

Elizabeth Patterson, MOccThySt, was Occupational Therapy Student, School of Health and Rehabilitation Sciences, The University of Queensland, St. Lucia, Brisbane, Queensland, Australia, at the time of the study.

Kathryn Marshall is Occupational Therapist, Department of Occupational Therapy, Princess Alexandra Hospital, Woolloongabba, Queensland, Australia.

Sally Bennett, PhD, is Associate Professor, School of Health and Rehabilitation Sciences, The University of Queensland, St. Lucia, Brisbane, Queensland, Australia.

Kylie Bower is Occupational Therapist, Department of Occupational Therapy, Princess Alexandra Hospital, Woolloongabba, Queensland, Australia.
OBJECTIVE. We explored the efficacy of compression gloves in maintaining the benefits gained from compression bandaging of the stroke-affected upper limb.

METHOD. Four participants completed a single-case $(A B C)$ design study consisting of a baseline period (Phase A) and compression bandaging of the hand and upper limb (Phase B), followed by the application of a compression glove (Phase $\mathrm{C}$ ). Edema was measured with circumferential tape at five specified points from the phalanx to the midforearm. All measurements were represented graphically for visual analysis, and celeration lines were calculated to indicate the degree of slope within each phase.

RESULTS. Visual analysis indicated fluctuating edema volume during Phase A, decreasing edema volume during Phase $B$, and a mixed trend during Phase $C$.

CONCLUSION. Compression gloves had mixed benefits in managing reductions in edema volume poststroke. Further research may consider the material, sizing, and style of glove in the development of a maintenance strategy.

Gustafsson, L., Patterson, E., Marshall, K., Bennett, S., \& Bower, K. (2016). Efficacy of compression gloves in maintaining edema reductions after application of compression bandaging to the stroke-affected upper limb. American Journal of Occupational Therapy, 70, 7002290030. http://dx.doi.org/10.5014/ajot.2016.017939

7 he loss of upper-extremity control is common after stroke, and approximately $80 \%$ of people with stroke experience upper-limb dysfunction (National Stroke Association, 2014). When people lose the ability to actively move their limb, an abnormal amount of fluid accumulates in the intercellular spaces in the tissues of the limb, leaving them susceptible to persistent edema (Roper, Redford, \& Tallis, 1999; Vasudevan \& Melvin, 1979). Reported incidence rates of edema of the upper limb have varied between studies and have ranged from $16 \%$ to $82.5 \%$ (Boomkamp-Koppen, Visser-Meily, Post, \& Prevo, 2005). Differences in definition and measurement have been proposed to account for the variance in reported incidence rates (Kuppens, Pijlman, Hitters, \& van Heugten, 2014). However, a recent study applied the same definition and measurement procedures at two data collection sites and reported incidence rates of $35 \%$ and $50 \%$, respectively, at these sites (Kuppens et al., 2014). The results imply that factors such as the treatment center and management protocols or perhaps the fluctuating and unpredictable nature of poststroke edema may be responsible for variation in incidence rates.

\section{Poststroke Upper-Limb Edema}

The etiology of poststroke upper-limb edema remains unclear. However, the physiological process has been linked to immobility, with changes in muscle tone 
and an impaired muscle pump disturbing the venous and lymphatic function (Dirette \& Hinojosa, 1994; ExtonSmith \& Crockett, 1957; Faghri, 1997; Leibovitz et al., 2007; Villeco, 2012). Villeco (2012) maintained that edema occurs when an imbalance of pressures is present across the capillary membrane. Capillary filtration pressure moves fluid from the capillaries outward into the tissues, and tissue hydrostatic pressure moves fluid from the tissue into the capillaries. Edema develops when an imbalance is present in these pressures and more fluid remains in the interstitial space (Howard \& Krishnagiri, 2001; Vasudevan \& Melvin, 1979; Villeco, 2012). Capillary filtration pressure and tissue hydrostatic pressure systems cannot function efficiently without joint movement (Petrek, Pressman, \& Smith, 2000; Vasudevan \& Melvin, 1979), thereby causing increased risk of edema developing and remaining in a stroke-affected upper limb.

Persistent edema can cause pain, stiffness, and fibrosis; in some chronic cases, persistent edema may predispose clients to contracture (Giudice, 1990; Hunter \& Mackin, 1984; Roper et al., 1999). These conditions may lead to an overall loss of function in the extremity that prevents clients from engaging in activities of daily living and diminishes their quality of life (Vasudevan \& Melvin, 1979). Rehabilitation may be impeded, and occupational therapists may observe that the client has diffculty participating in occupational roles and activities as a result of the edema.

\section{Compression Therapy for Poststroke Edema}

Compression therapy is the application of a circumferential pressure around the affected extremity with the purpose of reducing edema volume (Woods, 2007). Two singlecase-design studies have investigated the effect of different compression bandages in reducing edema of the strokeaffected hand (Gustafsson, Lunnon, Hoyle, Marshall, \& Bower, 2015; Gustafsson, Walter, Bower, Slaughter, \& Hoyle, 2014). In the first study, Gustafsson et al. (2014) examined whether low-stretch $(n=4)$ or high-stretch $(n=4)$ bandages were more effective in reducing edema in the stroke-affected hand. In the second study, Gustafsson et al. (2015) examined whether bandaging of the entire limb $(n=6)$ was more efficient than bandaging of the hand alone $(n=6)$. Both studies demonstrated that compression bandaging for a 7-day period was an effective intervention technique for the short-term reduction of edema in the stroke-affected hand in all participants. No clear difference was found between the low- and highstretch bandages, and a trend was seen for bandaging of the entire limb to result in greater reduction in edema in the forearm. Both studies demonstrated that the edema returned after removal of the bandages, which suggested that future research is needed to find a treatment option to maintain the reductions gained from bandaging.

In clinical practice, compression garments are used to maintain the reductions gained from compression bandaging in clients with lymphedema (Johansson, Albertsson, Ingvar, \& Ekdahl, 1999). A single cross-over case-control study by Gracies et al. (2000) assessed the efficacy of the application of a custom-made upper-limb spandex garment on a range of factors poststroke, including edema. The garment consisted of a custommade sleeve for the arm and a glove that were targeted at improving positioning of the upper limb. Six participants in the study had limb edema, and the results indicated a small yet significant reduction in swelling of the fingers and forearm after the 3-hr treatment period (Gracies et al., 2000). The results, although statistically significant, did not indicate a gain in long-term benefits from wearing the garment, and further studies are required to support the findings with this small sample.

A treatment center in the Netherlands has developed and trialed a protocol for the management of edema that includes cryotherapy, splinting, compression bandaging, and compression glove (Kuppens et al., 2014). The trial was designed to determine the incidence and duration of edema across two rehabilitation centers and to evaluate the impact of the edema protocol at one center. It was the first longitudinal study to follow people with edema in their stroke-affected upper limb during the entire inpatient rehabilitation period $(N=206)$ and $\leq 8 \mathrm{wk}$ after discharge $(N=128)$. The study did not demonstrate a treatment effect in favor of the protocol group, and the authors reported that group differences at baseline may have affected the results.

\section{Compression Garments}

The rationale for using compression to treat poststroke edema is based on its demonstrated effectiveness in the treatment of lymphedema. The application of compression garments to maintain the benefits gained from bandaging during the maintenance phase of lymphedema therapy has proven long-term efficacy (Bernas, Witte, \& Witte, 2001; Johansson et al., 1999; Zuther, 2009). Compression garments, which must be prescribed by the treating physician, are available in four classes: Classes 1 and 2 for lower compression and Classes 3 and 4 for higher compression (Zuther, 2009). Compression garments are further classified into two distinct knit types: 
circular and flat. Circular-knit garments are typically used for lower compression (Classes 1-2) in clients with early to moderate lymphedema (i.e., uniform swelling and $<20 \%$ excess fluid volume). For clients with more severe lymphedema (i.e., an excess fluid volume of $>20 \%$ ), flatknit garments with higher compression (Classes 3-4) are more suitable. These garments are also prescribed for clients with limb-shape distortion (Lymphoedema Support Network, 2012; Woods, 2007)

A common clinical practice in the area of stroke care is to have clients wear an edema glove that applies a lower level of compression after compression bandaging. Applying the principles outlined previously, poststroke edema is generally noted in the early phases and represents $<20 \%$ excess fluid volume; one may therefore presume that clients presenting with poststroke edema may respond better to circular-knit compression garments than to flat-knit garments (Woods, 2007). Studies evaluating the impact of compression bandages followed by compression garments for the stroke-affected upper limb have been limited. Therefore, this study contributes to the current body of evidence for edema management by exploring whether Class 2 circular-knit compression gloves retain the reduction in edema after the application of compression bandaging to the affected upper limb in people with stroke.

\section{Method}

A single-case-design study evaluated the use of compression gloves after treatment with compression bandaging for hand and forearm edema in 4 participants with poststroke edema. Approval for this study was received from hospital and university ethics committees. Informed written consent was obtained from the participants.

\section{Participants}

The participants were recruited from the rehabilitation unit, the acute stroke unit, or the internal medicine ward of a major metropolitan hospital. Inclusion criteria stated that the participant had to be medically stable and have the cognitive and communicative abilities to consent to treatment. Clinically evident edema of the hand and forearm, defined as observable swelling compared with the nonaffected hand and forearm, had to be present. The treating physicians gave medical consent for compression bandaging and the fitting of compression gloves on the basis of their patients' vascular and medical conditions.

\section{Instrumentation}

A precise protocol was developed to ensure the reliable measurement of hand and forearm circumference and volume. The same three research team members took measurements at the same time of day for each participant. Studies have indicated that circumferential measurements taken consistently by the same people have a high level of intrarater reliability (Jansen et al., 2010). Using edema measurement tape, the team members collected data on circumferential measurements at five specific points on the affected upper limb: midproximal phalanx of the third digit, the metacarphophalangeal (MCP) joints, the wrist at the ulnar styloid, and at consecutive increments of $10 \mathrm{~cm}$ above the styloid process up to $20 \mathrm{~cm}$. Interrater reliability has been established, with Cronbach's $\alpha$ s ranging from .962 to .990 (Gustafsson et al., 2014), which exceeds the minimal requirement for interrater reliability (Bravo \& Potvin, 1991).

\section{Intervention}

This was a pragmatic study that evaluated the best practice for edema management in the participating hospital center. Each participant received the same treatment: massage and elevation for 3 consecutive days, after which compression bandaging was applied for 7 days followed immediately by the application of a Class 2 circular-knit compression glove for 7 days. The circumferential compression bandaging was applied distally from the fingers to the axilla. The bandaging commenced on the ulnar side of the hand, wrapped twice around the MCPs with 50\% stretch, and then continued through the web space of the thumb and up the arm using 50\% overlap. A short stretch cohesive bandage was used for the fingers, and a low-stretch cohesive bandage was used for the palm and up the arm.

The compression glove used in this study was a Class 2 circular-knit compression glove, which was standard clinical practice in the treatment center. The glove can be purchased off the shelf and can immediately be applied to the affected hand. Circumferential measurements for the glove were taken at each finger, the thumb, and wrist. The size of the glove selected for each participant was dictated by the manufacturer's measurements, which corresponded to a glove size of small, medium, large, or extra large.

\section{Procedure}

The procedure for this study followed the measurement regimen of the previous studies (see Gustafsson et al., 2014, 2015).

Baseline Phase A. After participant recruitment, the therapist took baseline measurements of the participants' stroke-affected upper limb on 3 consecutive days. Standard clinical interventions of massage and elevation were conducted during the baseline period. 


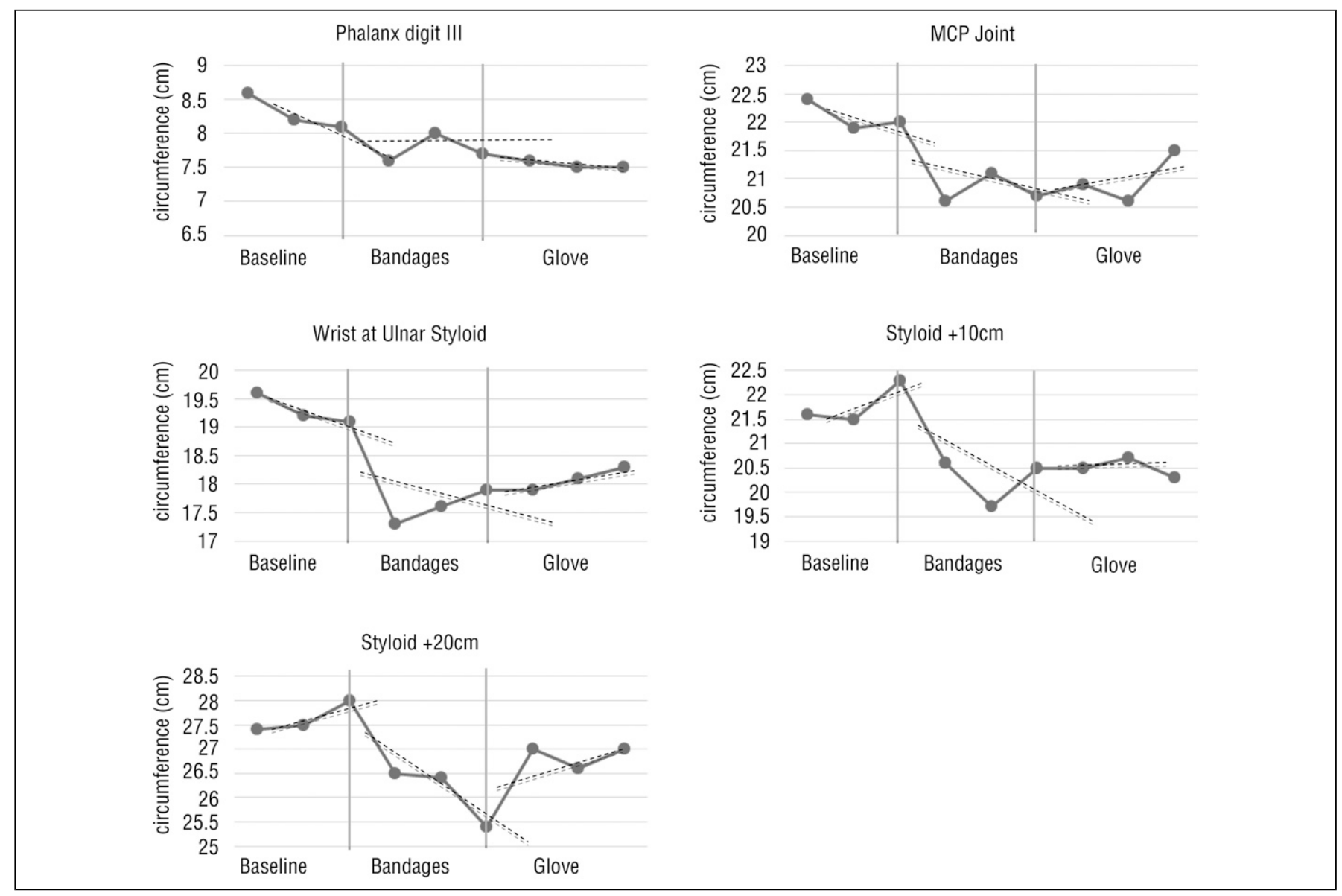

Figure 1. Participant 1.

Note. MCP $=$ metacarpophalangeal.

Intervention Phase B. Compression bandaging was applied to the upper limb immediately after the third baseline measurement. The participants wore the compression bandages for a total of 7 days, and the bandages were removed on three occasions to record circumferential measurements. The compression bandages were not reapplied after the third measurement on the 7 th day.

Intervention Phase C. A Class 2 circular-knit compression glove was applied to the stroke-affected hand immediately after removal of the compression bandages. The participants wore the gloves all day for a total of 7 days. The measurement protocol from Phase B was repeated, with three measurements at fixed intervals during the 7 days.

\section{Data Analysis}

Data analysis of single-case designs does not rely on statistical hypothesis testing of responses collected from a sample of participants. The standard method of data analysis in single-subject research is visual analysis of patient responses graphed over time (Bobrovitz \& Ottenbacher, 1998). Data for each measurement point were plotted on a graph and analyzed visually to determine the effects of the intervention. The graphs show the circumferential measurement points at each of the five anatomical positions for each participant. The data were split into three sections to delineate the different phases: baseline (A), bandages (B), and glove (C). Data within each phase were analyzed visually and by using celeration lines, which indicated the trend of the data. To draw the celeration line, data were taken from within each phase at the $x$-axis and split in half, halving the data again to find the median. The celeration line was drawn by connecting the median points within each phase (Portney \& Watkins, 2009). The rate of change was determined by visually analyzing the stability, trend, and level of the celeration line within and between each phase (Portney \& Watkins, 2009).

\section{Results}

Four participants were recruited to the study, with time from stroke onset ranging from 35 to 82 days. No participants had active movement return; 3 had the left upper limb affected, and 1 had the right upper limb affected. Three participants had soft edema, and 1 participant had 


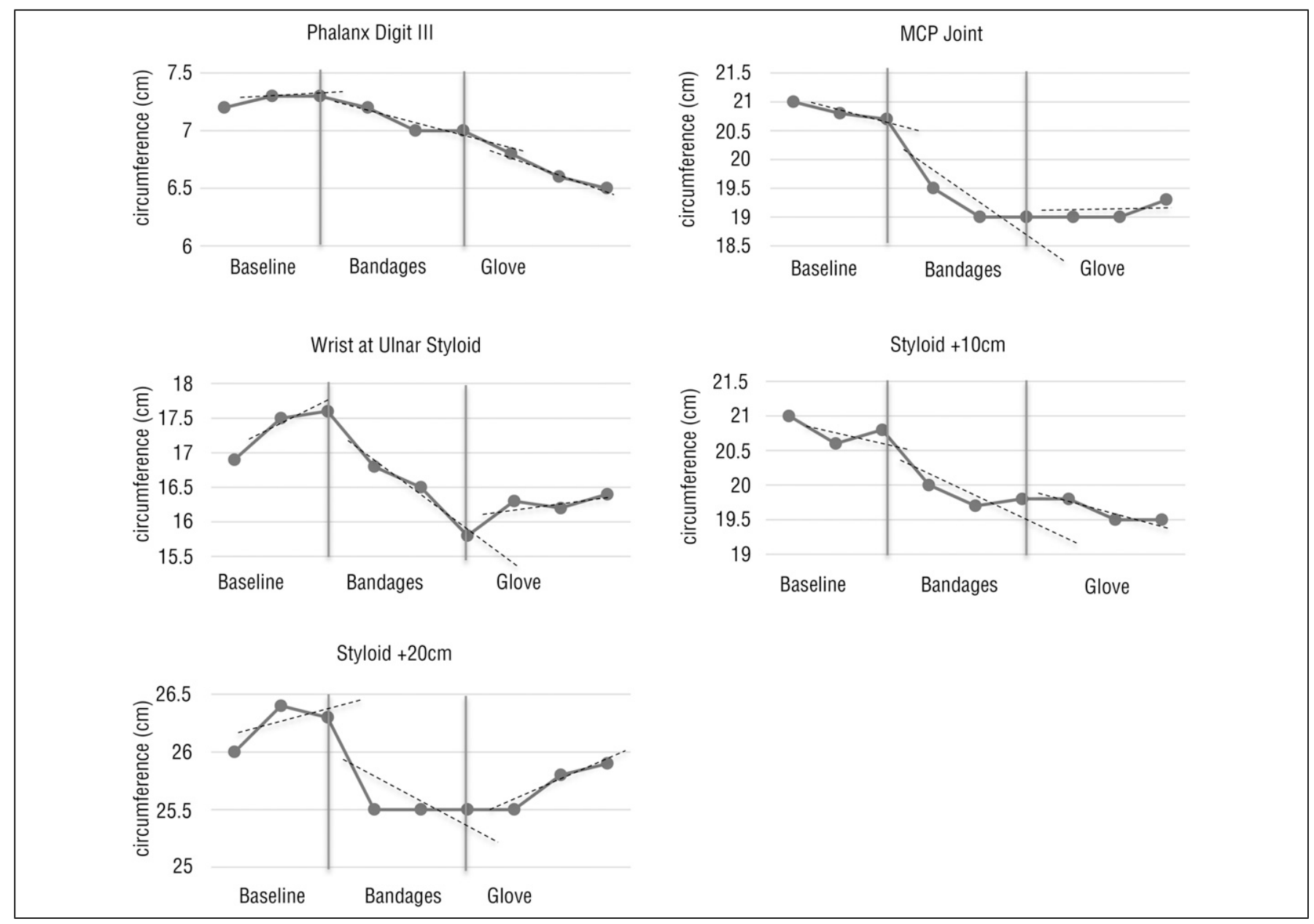

Figure 2. Participant 2.

Note. MCP $=$ metacarpophalangeal.

firm or fibrous edema. Two participants demonstrated some hypertonicity in their fingers. Figures 1-4 illustrate the data for the 4 participants, with celeration lines shown for each phase.

\section{Within-Phase Analysis}

Fluctuations occurred in the data in each phase, but there was a general trend as evidenced by the celeration lines. During Phase A, a mixed trend was shown for increasing or decreasing volume. In Phase B, a consistent trend across all anatomical points indicated a decrease in volume. During Phase C, the trend was for increasing volume at all anatomical points except the phalanx.

\section{Between-Phases Analysis}

Changes in the celeration line trend and level indicated that the compression bandages used during Phase B were generally effective in reducing edema volume. The decrease in volume was evident across all measurement points for all participants. The reductions in edema recorded during Phase B were retained at the phalanx for all participants, with a trend toward increasing edema at all other measurement points. There was a trend for measurements to not reach baseline levels, with the exceptions of Participants 3 and 4 at the styloid $+20-\mathrm{cm}$ measurement point.

\section{Discussion}

The aim of this study was to examine whether a compression glove maintained the benefits gained from compression bandaging of the stroke-affected hand and arm. Compression gloves are used to treat poststroke edema in the clinical setting predominantly on the basis of the evidence supporting the use of compression garments to treat lymphedema (Lymphoedema Support Network, 2012; Petrek et al., 2000). Previous studies (Gustafsson et al., 2014, 2015) have demonstrated that compression bandaging reduces hand and arm edema poststroke but that the edema returns after removal of the bandaging. This study followed the protocol for lymphedema management by first using compression bandaging to reduce edema and then administering the compression glove to maintain the reduction in edema 


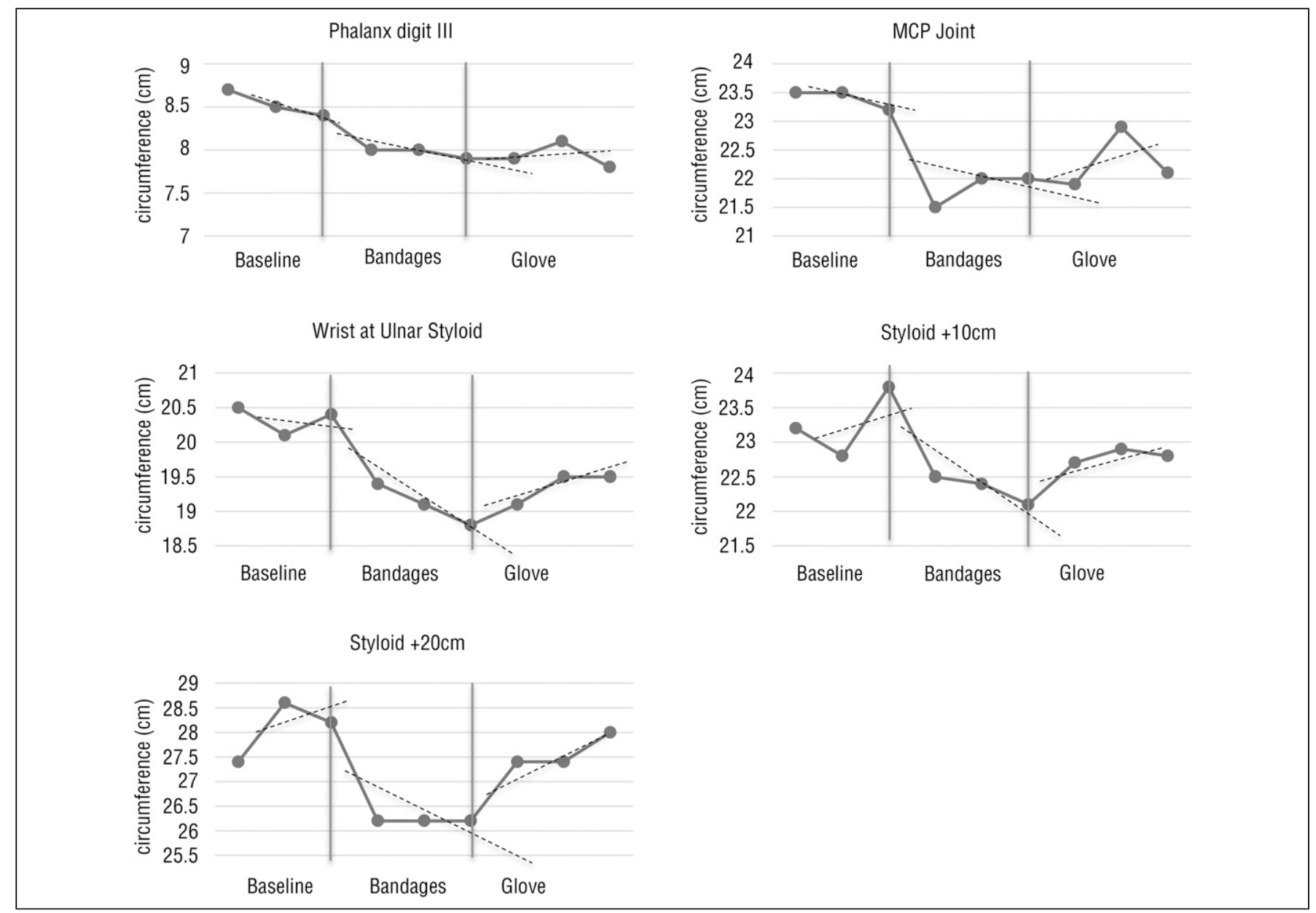

Figure 3. Participant 3.

Note. MCP $=$ metacarpophalangeal.

levels (Bernas et al., 2001; Lymphoedema Support Network, 2012; Petrek et al., 2000; Woods, 2007). Consistent with previous results, we found a general instability in edema levels with a tendency for daily fluctuations and a decreasing trend (i.e., increased rate of decrease) in edema measures after bandaging was applied (Gustafsson et al., 2014, 2015). However, the focus of this study was the maintenance effects of the compression glove. The results suggest that although edema volume did not return to preintervention levels, the glove did not successfully maintain the total benefits gained from the compression bandages.

Several factors require further exploration. First, the trend for maintenance or continuing reduction in the phalanx measurements and not in the MCP or wrist measurements suggests that effectiveness may have been affected by reduced fit of the glove. Unlike the custom garments used by Gracies et al. (2000), the compression glove in this study was an off-the-shelf product, with standard sizing that makes it readily accessible in a clinical setting. To optimize results in future studies, each participant may require a custom-made compression glove designed to fit his or her specific measurements. Second, the return of the edema to the forearm and wrist suggest that a compression glove in isolation may not be sufficient and that the compression garment should extend further up the forearm, which may have significant implications for clinical practice because the compression garments in the Gracies et al. study incorporated the forearm and the upper arm.

A final consideration relates to assumptions made about the mechanism of the upper-limb edema that link lymphedema practices to poststroke edema. Typically, circular-knit garments with pressure ranging from Class 1 to Class 2 are prescribed to people with upper-limb lymphedema (Woods, 2007). Circular-knit gloves tend to be thin in texture and can be bought over the counter. Because of the immediate availability of this type of glove and its use in upper-limb lymphedema, these gloves were used in the study treatment center. However, poststroke edema often presents in combination with hemiplegia of the upper limb, and this additional loss of muscle action and muscle pump may require a different type of glove. An alternative type of compression glove may have had 


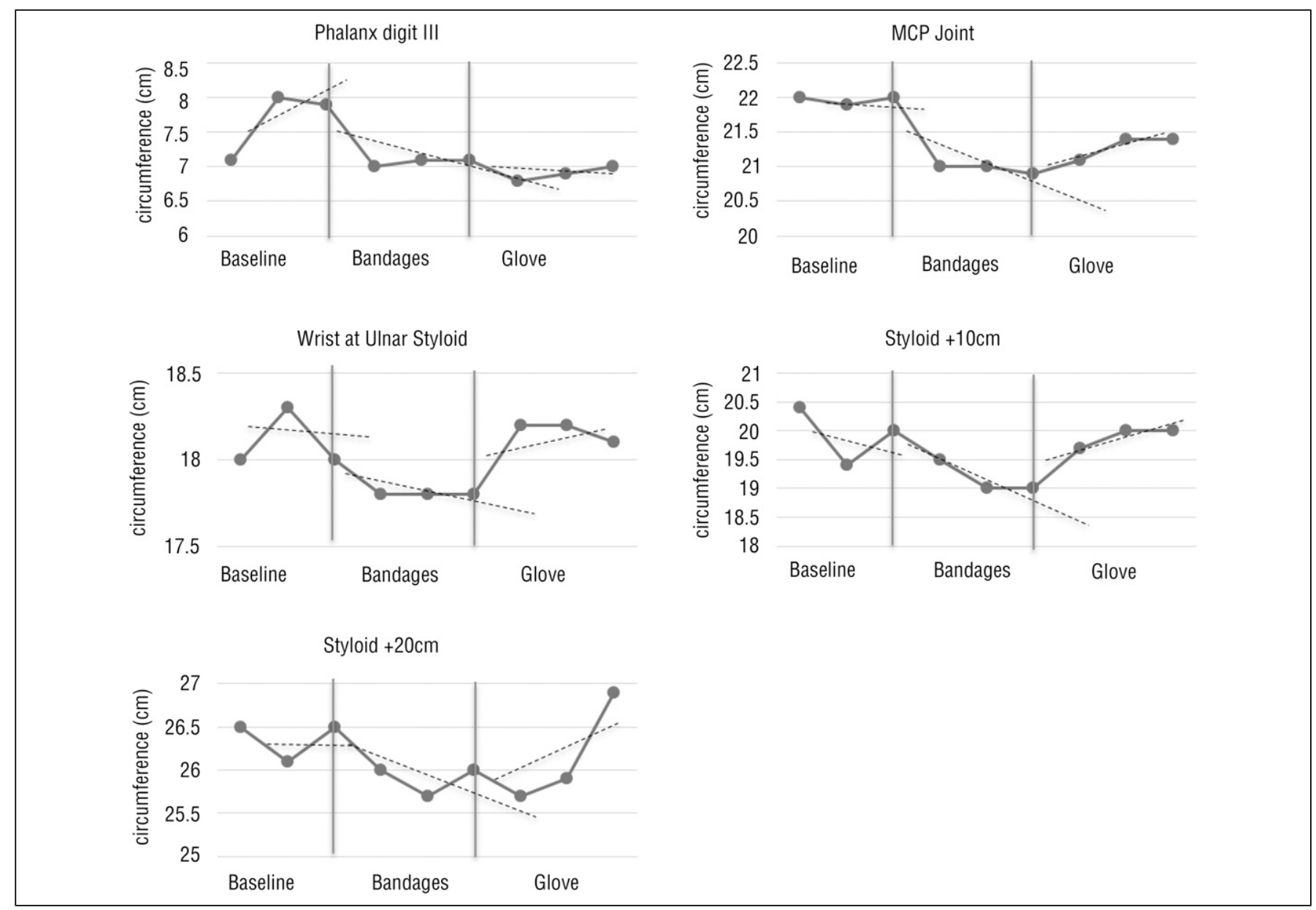

Figure 4. Participant 4.

Note. MCP = metacarpophalangeal.

better results among participants in this study. Flat-knit compression gloves are also used for people with lymphedema who require increased circumferential pressure (Classes 3-4). The flat-knit glove material is more expensive, thicker, and less elastic than the circular-knit glove material and can only be custom made to fit the patient (Woods, 2007). The dynamic spandex splints used in the study by Gracies et al. (2000) were likely fabricated from a flat-knit compression garment because of the force required to effect the changes in positioning that were the focus of that study. Future studies should carefully consider the type of garment that is trialed, including knit, length, and type of fit.

This study was designed as an exploratory study and, therefore, because of the small sample size and the singlestudy research design, the study results do not allow for strong conclusions to be drawn. Future research studies would benefit from incorporating larger samples of participants and multiple types of garments (i.e., spandex, flat knit, custom made) with varying degrees of pressure (Classes 1-4) and lengths up the arm. Moreover, the study was completed at one treatment center and in a 15-day protocol that may have limited the treatment effects for individual participants. Future research would benefit from maintaining the compression bandaging for as many days as necessary until edema has been removed from the limb. A combination of these recommendations would provide clinicians with evidence to make stronger conclusions about the use of compression garments to treat hand and forearm edema poststroke.

\section{Implications for Occupational Therapy Practice}

The implications of this study for occupational therapy practice are as follows:

- The use of compression bandages seems effective in decreasing edema after stroke, but whether compression gloves can retain the reductions achieved remains uncertain.

- Further research is required to identify the most effective regimen to manage edema. Consideration of the material, the sizing, and the style of glove will be important factors when developing a maintenance strategy. 


\section{Conclusion}

The results of this study indicate that the Class 2 circular compression glove had mixed benefits in maintaining reductions in edema achieved with initial compression bandaging of the hand and arm after stroke. The findings are not consistent with evidence from the lymphedema research on which this study was based. This inconsistency may be due to the fluctuating and unique nature of edema in the hand and arm after stroke, or it may demonstrate a need to identify gloves that are applicable to poststroke edema. Further research is required to determine the optimal protocol and glove and to develop the evidence base for this area of practice.

\section{Acknowledgments}

This work was partially supported by the National Stroke Foundation (Honours Research Grant 2012). We gratefully acknowledge the support of Crystal Robertson and Katrina Whybird and the clients involved in the study. The authors declare that there is no conflict of interest.

\section{References}

Bernas, M. J., Witte, C. L., \& Witte, M. H.; International Society of Lymphology Executive Committee. (2001). The diagnosis and treatment of peripheral lymphedema: Draft revision of the 1995 Consensus Document of the International Society of Lymphology Executive Committee for discussion at the September 3-7, 2001, XVIII International Congress of Lymphology in Genoa, Italy. Lymphology, 34, 84-91.

Bobrovitz, C. D., \& Ottenbacher, K. J. (1998). Comparison of visual inspection and statistical analysis of single subject data in rehabilitation research. American Journal of Physical Medicine and Rehabilitation, 77, 94-102.

Boomkamp-Koppen, H. G., Visser-Meily, J. M., Post, M. W., \& Prevo, A. J. (2005). Poststroke hand swelling and oedema: Prevalence and relationship with impairment and disability. Clinical Rehabilitation, 19, 552-559. http://dx.doi.org/ $10.1191 / 0269215505 \mathrm{cr} 8460 \mathrm{a}$

Bravo, G., \& Potvin, L. (1991). Estimating the reliability of continuous measures with Cronbach's alpha or the intraclass correlation coefficient: Toward the integration of two traditions. Journal of Clinical Epidemiology, 44, 381-390. http://dx.doi.org/10.1016/0895-4356(91)90076-L

Dirette, D., \& Hinojosa, J. (1994). Effects of continuous passive motion on the edematous hands of two persons with flaccid hemiplegia. American Journal of Occupational Therapy, 48, 403-409. http://dx.doi.org/10.5014/ajot.48.5.403

Exton-Smith, A. N., \& Crockett, D. J. (1957). Nature of oedema in paralysed limbs of hemiplegic patients. British Medical Journal, 2, 1280-1283. http://dx.doi.org/10.1136/ bmj.2.5056.1280

Faghri, P. D. (1997). The effects of neuromuscular stimulation-induced muscle contraction versus elevation on hand edema in CVA patients. Journal of Hand Therapy, 10, 29-34. http://dx.doi.org/10.1016/S0894-1130(97)80008-7

Giudice, M. L. (1990). Effects of continuous passive motion and elevation on hand edema. American Journal of Occupational Therapy, 44, 914-921. http://dx.doi.org/10.5014/ ajot.44.10.914

Gracies, J. M., Marosszeky, J. E., Renton, R., Sandanam, J., Gandevia, S. C., \& Burke, D. (2000). Short-term effects of dynamic Lycra splints on upper limb in hemiplegic patients. Archives of Physical Medicine and Rehabilitation, 81, 1547-1555. http://dx.doi.org/10.1053/apmr.2000.16346

Gustafsson, L., Lunnon, J., Hoyle, M., Marshall, K., \& Bower, K. (2015). What method of compression bandaging is more effective for post-stroke oedema of the upper limb? Manuscript submitted for publication.

Gustafsson, L., Walter, A., Bower, K., Slaughter, A., \& Hoyle, M. (2014). Single-case-design evaluation of compression therapy for edema of the stroke-affected hand. American Journal of Occupational Therapy, 68, 203-211. http://dx.doi.org/ 10.5014/ajot.2014.009423

Howard, S. B., \& Krishnagiri, S. (2001). The use of manual edema mobilization for the reduction of persistent edema in the upper limb. Journal of Hand Therapy, 14, 291-301. http://dx.doi.org/10.1016/S0894-1130(01)80008-9

Hunter, J. M., \& Mackin, E. J. (1984). Edema and bandaging. In J. M. Hunter, L. H. Schneider, E. J. Mackin, \& A. D. Callahan (Eds.), Rehabilitation of the hand (2nd ed., Vol. 1, pp. 146-153). St. Louis, MO: C. V. Mosby.

Jansen, V., Radbourne, L., Fakis, A., Bradley, M., Burke, F., \& Ellis, J. (2010). Validity, responsiveness, intra- and interrater reliability of the weighted tape measure when measuring digital circumference. Hand Therapy, 15, 31-38. http://dx.doi.org/10.1258/ht.2010.010009

Johansson, K., Albertsson, M., Ingvar, C., \& Ekdahl, C. (1999). Effects of compression bandaging with or without manual lymph drainage treatment in patients with postoperative arm lymphedema. Lymphology, 32, 103-110.

Kuppens, S. P., Pijlman, H. C., Hitters, M. W., \& van Heugten, C. M. (2014). Prevention and treatment of hand oedema after stroke. Disability and Rehabilitation, 36, 900-906. http://dx.doi.org/10.3109/09638288.2013.824031

Leibovitz, A., Baumoehl, Y., Roginsky, Y., Glick, Z., Habot, B., \& Segal, R. (2007). Edema of the paretic hand in elderly post-stroke nursing patients. Archives of Gerontology and Geriatrics, 44, 37-42. http://dx.doi.org/10.1016/ j.archger.2006.02.005

Lymphoedema Support Network. (2012). The use of compression garments in lymphoedema management. Retrieved March 1, 2014, from http:/www.lymphoedema.org/ Menu2/Index.asp

National Stroke Association. (2014). Hemiparesis. Retrieved March 1, 2014, from http://www.stroke.org/site/PageServer? pagename $=$ hemiparesis

Petrek, J. A., Pressman, P. I., \& Smith, R. A. (2000). Lymphedema: Current issues in research and management. CA: A Cancer Journal for Clinicians, 50, 292-307, quiz 308-311.

Portney, L. G., \& Watkins, M. P. (2009). Foundations of clinical research: Applications to practice (3rd ed.). Upper Saddle River, NJ: Prentice Hall. 
Roper, T. A., Redford, S., \& Tallis, R. C. (1999). Intermittent compression for the treatment of the oedematous hand in hemiplegic stroke: A randomized controlled trial. Age and Ageing, 28, 9-13. http://dx.doi.org/10.1093/ageing/ 28.1.9

Vasudevan, S. V., \& Melvin, J. L. (1979). Upper extremity edema control: Rationale of the techniques. American Journal of Occupational Therapy, 33, 520-523.
Villeco, J. P. (2012). Edema: A silent but important factor. Journal of Hand Therapy, 25, 153-161, quiz 162. http:// dx.doi.org/10.1016/j.jht.2011.09.008

Woods, M. E. (2007). Lymphoedema care. Oxford, England: Blackwell. http://dx.doi.org/10.1002/9780470692158

Zuther, S. (2009). Complete decongestive therapy. In J. E. Zuther (Ed.), Lymphedema management: The comprehensive guide for practitioners (2nd ed., p. 122). New York: Thieme. 
Reproduced with permission of the copyright owner. Further reproduction prohibited without permission. 